\title{
Apolipoprotein E polymorphism in Japanese patients with Alzheimer's disease or vascular dementia
}

\author{
Jun Kawamata, Seigo Tanaka, Shun Shimohama, Kunihiro Ueda, Jun Kimura
}

\begin{abstract}
Apolipoprotein E (ApoE) plays a key part in lipid metabolism both in the liver, and in the CNS. To clarify the association of ApoE polymorphism with Alzheimer's disease and vascular dementia in Japan, 13 patients with early onset (age $\geqslant 65$ ) sporadic Alzheimer's disease, 40 patients with late onset (age $\leqslant 65$ ) sporadic Alzheimer's disease, 19 patients with vascular dementia, and 49 non-demented control subjects were analysed. The results showed a significantly increased frequency of the $\varepsilon 4$ allele in the patients with late onset sporadic Alzheimer's disease $(0 \cdot 25)$, but not in the patients with early onset sporadic Alzheimer's disease $(0.04)$ or in the patients with vascular dementia $(0 \cdot 13)$ compared with controls $(0 \cdot 09)$. The raised frequency of the $\varepsilon 4$ allele in the patients with late onset sporadic Alzheimer's disease was of a lower magnitude than that in United States and Canadian studies. This may in part be due to a lower $\varepsilon 4$ frequency in the normal Japanese population and reflect the lower morbidity from Alzheimer's disease in Japan.
\end{abstract}

(F Neurol Neurosurg Psychiatry 1994;57:1414-1416)

Faculty of Medicine, Kyoto University, Kyoto, Japan

Department of Neurology

J Kawamata

S Shimohama

J Kimura

Department of Clinical Science and Laboratory Medicine $S$ Tanaka

K Ueda

Correspondence to: Dr Shun Shimohama Dr Shrt Shama, Deparment of Neurology, Faculty of Medicine, K University, 54 Shogoin Kyoto 606, Japan.

Received 7 February 1994 and in final revised form 10 May 1994 Accepted 24 May 1994
Apolipoprotein E (ApoE) is a structural component of chylomicrons, very low, intermediate, and high density lipoproteins. ${ }^{1}$ It plays key parts in the metabolism of cholesterol and triglyceride in plasma through the uptake of ApoE containing lipoprotein by low density lipoprotein (LDL) receptors or by the LDLreceptor related protein (LRP) in liver. Although ApoE is mainly synthesised in the liver, it is also synthesised in the CNS, and is a major apolipoprotein in the CSF. ${ }^{1}$ Recently ApoE and LRP immunoreactivities have been found to be associated with the amyloid of senile plaques, vascular amyloid, and neurofibrillary tangles in the brains of patients with Alzheimer's disease. ${ }^{23}$ Moreover, several reports indicate that $\mathrm{ApoE}$ binds strongly to synthetic $\beta / \mathrm{A} 4$ peptide. ${ }^{4}$ These findings have led to a hypothesis that ApoE- $\beta / \mathrm{A} 4$ complexes may be taken up by LRP on activated astrocytes or neurons, mediating clearance of $\beta / \mathrm{A} 4$ from neurophils. ${ }^{3}$

ApoE has three major isoforms, referred to as ApoE2, ApoE3, and ApoE4, which are encoded by three alleles, $\varepsilon 2, \varepsilon 3$, and $\varepsilon 4$, respectively, at a single locus on the long arm of chromosome 19. Recently, Strittmatter et $a l^{4}$ reported an increased frequency of the $\varepsilon 4$ allele in late onset familial and sporadic Alzheimer's disease in the United States. Increased frequency of the $\varepsilon 4$ allele in Japanese patients with multi-infarct dementia was also reported. ${ }^{6}$

To clarify further the relation between ApoE polymorphism and dementia, we analysed the genotype of ApoE in Japanese patients with early onset (age $\leqslant 65$ ) and late onset (age $>65$ ) sporadic Alzheimer's disease, vascular dementia, and Japanese nondemented controls.

\section{Patients and methods}

DNA was extracted from leucocytes of 13 patients with early onset sporadic Alzheimer's disease, 40 patients with late onset sporadic Alzheimer's disease, 19 patients with vascular dementia, and 49 non-demented subjects. All the patients and normal subjects were residents of Kyoto city and its suburbs. Alzheimer's disease was diagnosed clinically as probable Alzheimer's disease on the basis of the NINCDS-ADRDA criteria. ${ }^{7}$ Patients with sporadic Alzheimer's disease were defined as those without a known family history of Alzheimer's disease or dementia. Vascular dementia was diagnosed clinically as probable vascular dementia and classified as "small vessel disease with dementia" on the basis of the NINDS-AIREN criteria. ${ }^{8}$ Patients with a history of diabetes mellitus, hyperlipidaemia, or other severe complications were excluded from analysis in this study. The average age of onset of dementia of the patients with early onset sporadic Alzheimer's disease, late onset sporadic Alzheimer's disease, and vascular dementia were (mean (SD)) 56.3 (6.5), 77.9 (6.8) and $74.5(6.9)$ years respectively. The mean age of control subjects at examination was $57 \cdot 1(18 \cdot 4)$ with a range of $26-90$ years. The 
control subjects were matched with each group of patients for age.

ApoE genotyping was performed with the polymerase chain reaction-restriction fragment length polymorphism method. Briefly, genomic DNA was amplified by the polymerase chain reaction in a DNA thermal cycler (Perkin Elmer Cetus) with an upstream primer: 5'-TCCAAGGAGCTGCAGGCGGCGCA-3' and a downstream primer: 5'-ACAGAATTCGCCCCGGCCTGGTACACTG-3'. Each amplification reactor contained $200 \mathrm{ng}$ of genomic DNA, 6 pmol of each primer, $5 \mathrm{nmol}$ of each dNTP, the supplied buffer (Cetus), and 0.6 units Taq DNA polymerase (Amplitaq, Cetus) in $25 \mu \mathrm{l}$. An initial denaturation at $95^{\circ} \mathrm{C}$ for three minutes was followed by 30 cycles of annealing at $55^{\circ} \mathrm{C}$ for one minute, extension at $72^{\circ} \mathrm{C}$ for two minutes, denaturation at $95^{\circ} \mathrm{C}$ for one minute, and a final extension at $72^{\circ} \mathrm{C}$ for five minutes. After at least three hours of digestion at $37^{\circ} \mathrm{C}$ with 10 units $\mathrm{CfoI}$ (Boehringer), each reaction mixture was loaded on a $4 \%$ agarose gel (NuSieve, FMC) and treated by electrophoresis for two hours at $100 \mathrm{~V}$, then photographed under UV light after staining with ethidium bromide. A $\chi^{2}$ test was used to examine the significance of the polymorphic difference between the groups.

\section{Results}

Tables 1 and 2 show the prevalence of each genotype and the allele frequencies. The overall frequencies of alleles $\varepsilon 2, \varepsilon 3$, and $\varepsilon 4$ were $0.000,0.962$, and 0.039 , respectively, in the early onset Alzheimer's disease group; $0.025,0.725$, and 0.250 in the late onset Alzheimer's disease group; $0.053,0.816$, and 0.132 in the vascular dementia group; and $0 \cdot 102,0.806$, and 0.092 in the control group.

There is no significant difference between the allele frequency of control subjects in this report and that reported previously ${ }^{9}$ for the normal Japanese population.

The frequency of the $\varepsilon 4$ allele was significantly increased in patients with late onset sporadic Alzheimer's disease $\left(p<0.01, \chi^{2}\right.$ test), but not in those with early onset sporadic Alzheimer's disease compared with that of the present control subjects or that reported previously for the normal Japanese population. ${ }^{9}$ The mean age of onset for patients with late onset sporadic Alzheimer's disease who had at least one $\varepsilon 4$ allele was less than that of the patients without the $\varepsilon 4$ allele $(75 \cdot 3(7 \cdot 2) v 79 \cdot 5(6 \cdot 2))$.

Table 1 Genotypes in patients with early onset sporadic Alzheimer's disease, late onset sporadic Alzheimer's disease, vascular dementia, and control subjects

\begin{tabular}{lllllll}
\hline Group (n) & $2 / 2$ & $2 / 3$ & $2 / 4$ & $3 / 3$ & $3 / 4$ & $4 / 4$ \\
\hline Alzheimer's disease: & & & & & & \\
$\quad$ Early onset (13) & 0 & 0 & 0 & 12 & 1 & 0 \\
Late onset (40) & 0 & 1 & 1 & 23 & 11 & 4 \\
Vascular dementia (19) & 0 & 2 & 0 & 13 & 3 & 1 \\
Control (49) & 0 & 9 & 1 & 31 & 8 & 0 \\
\hline
\end{tabular}

The frequency of the $\varepsilon 4$ allele in vascular dementia was slightly greater than the controls $(0.132 v 0.092)$, but the difference was not significant. The mean age of onset of patients with vascular dementia who had at least one $\varepsilon 4$ allele was also less than that of patients who had no $\varepsilon 4$ allele $(70 \cdot 0(9 \cdot 0) v$ $75 \cdot 7(6 \cdot 0))$.

\section{Discussion}

Our present results confirmed the high frequency of the $\varepsilon 4$ allele in patients with late onset sporadic Alzheimer's disease ( $p<0.01$, $\chi^{2}$ test), but not in patients with early onset sporadic Alzheimer's disease compared with non-demented controls. In late onset sporadic Alzheimer's disease, raised frequencies of the $\varepsilon 4$ allele as high as from 0.38 to 0.40 have been reported in United States ${ }^{5}$ and Canadian studies. ${ }^{10}$ Whereas, in the Japanese, it was found to be 0.25 in the present study (table 2). This lower figure is supposed to be due to the ethnic and environmental backgrounds. A previous report ${ }^{1}$ has shown that the frequency of the $\varepsilon 4$ allele in a normal Japanese population is lower than that in Caucasians $(0.11 v 0.16 ; \mathrm{p}<0.001)$. This difference may be one of the causes of the lower morbidity from Alzheimer's disease among the Japanese. Although we could not find an increased frequency of the $\varepsilon 4$ allele in early onset sporadic Alzheimer's disease, the mean age of onset of patients with late onset sporadic Alzheimer's disease who had at least one $\varepsilon 4$ allele was lower than that of the patients without the $\varepsilon 4$ allele $(75 \cdot 3(7 \cdot 2) v$ $79.5(6 \cdot 2))$. ApoE4 may act as an accelerating modulator in the course of late onset sporadic Alzheimer's disease.

As ApoE4 is reported to be a risk factor of coronary artery disease, ${ }^{1}$ similar pathophysiological effects may exist in the aetiology of vascular dementia..$^{6112}$ It is virtually impossible to distinguish mixed dementia or "Alzheimer's disease with cerebrovascular disease" based on NINDS-AIREN criteria from the so called vascular dementia strictly by clinical features and imaging. An increased frequency of the $\varepsilon 4$ allele in vascular dementia may be, to some extent, due to inevitable contamination with mixed dementia. To minimise these effects, we chose patients with vascular dementia who were classified as "small-vessel disease with dementia" by NINDS-AIREN criteria, and excluded patients whose CT findings showed moderate to severe cortical atrophy especially in the frontotemporal lobes. We also excluded patients with a history of diabetes mellitus, hyperlipidaemia, or other severe complications. As a result, the frequency of the $\varepsilon 4$ allele in vascular dementia was slightly increased compared with controls $(0.132 v$ 0.092 ), but the difference was not statistically significant. A previous Japanese report ${ }^{6}$ showed a higher $\varepsilon 4$ frequency $(0 \cdot 208)$ in patients with multi-infarct dementia. The mean age of onset of patients with vascular dementia who had at least one $\varepsilon 4$ allele was 
Table 2 Allele frequency of ApoE in patients with early onset sporadic Alzheimer's disease, late onset sporadic Alzheimer's disease, vascular dementia, and control subjects

\begin{tabular}{llll}
\hline Group $(n)$ & $\varepsilon 2$ & $\varepsilon 3$ & $\varepsilon 4$ \\
\hline Alzheimer's disease: & & & \\
$\quad$ Early onset (26) & $0.000(0)$ & $0.962(25)$ & $0.039(1)$ \\
Late onset (80) & $0.025(2)$ & $0.725(58)$ & $0.250(20)^{\star}$ \\
Vascular dementia (38) & $0.053(2)$ & $0.816(31)$ & $0.132(5)$ \\
Control (98) & $0.102(10)$ & $0.806(79)$ & $0.092(9)$ \\
Japanese normal population (638) & 0.081 & 0.849 & 0.067 \\
\hline
\end{tabular}

${ }^{\star} \mathrm{p}<0.01\left(\chi^{2}\right.$ test); late onset Alzheimer's disease $v$ control.

Allele numbers are in parentheses.
This work was supported by Grants-in-Aid for Scientific Research on Priority Areas (05261206) and for General Scientific Research $(04404043,05670557)$ from the Ministry of Education, Science and Culture, Japan.

1 Davignon J, Gregg RE, Sing CF. Apolipoprotein E polymorphism and atherosclerosis. Arteriosclerosis 1988;8: 1-21.

2 Namba $Y$, Tomonaga $M$, Kawasaki H, Otomo E, Ikeda $\mathrm{K}$. Apolipoprotein $\mathrm{E}$ immunoreactivity in cerebral amyloid deposits and neurofibrillary tangles in Alzheimer' disease and kuru plaque amyloid in Creutzfeldt-Jakob disease. Brain Res 1991;541:163-6.

3 Rebeck GM, Reiter JS, Strickland DK, Hyman BT Apolipoprotein $\mathrm{E}$ in the sporadic Alzheimer's disease allelic variation and receptor interactions. Neuron 1993 11:575-80.

also lower than that of those who had no $\varepsilon 4$ allele $(70.0 v 75 \cdot 7)$. These findings may only reflect the difficulty in diagnosing vascular dementia, but a possibility exists that ApoE4 may act as an accelerating modulator in the course of vascular dementia. High frequency of the $\varepsilon 4$ allele in ischaemic cerebrovascular disease was reported in one study, ${ }^{11}$ but in another study the frequency of the $\varepsilon 2$ allele was increased but not that of the $\varepsilon 4$ allele. ${ }^{12}$ This difference is thought to be due to the selection of patients.

In conclusion, the frequency of the $\varepsilon 4$ allele in patients with late onset sporadic Alzheimer's disease in Japan is increased, but not as much as previously reported in the United States and Canada, which may be due partly to a lower $\varepsilon 4$ allele frequency in the normal Japanese population than in Caucasians, and reflect the relatively lower morbidity from Alzheimer's disease in Japan. Genotypes of ApoE in patients with early onset sporadic Alzheimer's disease and vascular dementia were also evaluated, but no statistical difference from the controls could be found.
4 Strittmatter WJ, Saunders AM, Schmechel D, et al. Apolipoprotein E: High-avidity binding to $\beta$-amyloid and increased frequency of type 4 allele in late-onset familial Alzheimer disease. Proc Natl Acad Sci USA 1993;90:1988-91.

5 Saunders AM, Strittmatter WJ, Schmechel D, et al. Association of apolipoprotein $\mathrm{E}$ allele $\varepsilon 4$ with late-onset familial and sporadic Alzheimer's disease. Neurology 1993;43:1467-72.

6 Shimano $\mathrm{H}$, Ishibashi S, Murase $T$, Gotohda $T$, Yamada N, Takaku F, Ohtomo E. Plasma apolipoproteins in N, Takaku F, Ohtomo E. Plasma apolipoproteins in patients with $1989 ; 79: 257-60$.

7 McKhann G, Drachman D, Folstein M, Katzman R Price D, Stadlan EM. Clinical diagnosis of Alzheimer's under the auspices of Department of Health and Human Services Task Force on Alzheimer's disease.

8 Román GC, Tatemichi TK, Erkinjuntti T, et al. Vascular dementia: diagnostic criteria for research studies (Report of the NINDS-AIREN International Workshop). Neurology 1993;43:250-60.

9 Utermann G. Apolipoprotein E polymorphism in health and disease. Am Heart $\mathcal{F}$ 1987;113:433-40.

10 Poirier J, Davignon J, Bouthillier D, Kogan S, Bertrand P, Gauthier S. Apolipoprotein $\mathrm{E}$ polymorphism and Gauthier S. Apolipoprotein E polymorph

11 Pedro-Botet J, Sentí $M$, Nogués X, et al. Lipoprotein and apolipoprotein profile in men with ischemic stroke. Roles of lipoprotein(a), triglyceride-rich lipoproteins, and apolipoprotein E polymorphism. Stroke 1992;23: 1556-62.

12 Couderc R, Mahieux F, Bailleul S, Fenelon G, Mary R, Fermanian J. Prevalence of apolipoprotein E phenotype in ischemic cerebrovascular disease. A case-contro disease: report of the NINCDS-ADRDA Work Grou Neurology 1984;34:939-44. study. Stroke 1993;24:661-4. 